\title{
Treatment Outcomes Among Black Adults Receiving Medication for Opioid Use Disorder
}

\author{
Anna Beth Parlier-Ahmad ${ }^{1}$ (D) - Mickeal Pugh Jr ${ }^{1} \cdot$ Caitlin E. Martin ${ }^{2}$ \\ Received: 24 February 2021 / Revised: 23 June 2021 / Accepted: 24 June 2021 / Published online: 12 July 2021 \\ (C) W. Montague Cobb-NMA Health Institute 2021
}

\begin{abstract}
Largely due to structural racism, Black people with substance use disorder have worse outcomes than their White counterparts. The opioid epidemic has amplified these racial disparities. Little is known about strengths that buffer against the systemic issues that disproportionately impact Black adults with opioid use disorder (OUD), particularly those receiving buprenorphine for OUD. The objectives of this study are to (1) assess psychosocial and clinical predictors of OUD outcomes and (2) explore differences in OUD outcomes by gender among a sample of Black adults receiving buprenorphine. This is a secondary data analysis of a cross-sectional survey and medical record review with a convenience sample recruited from an addiction medicine clinic. Analyses included Black participants who provided at least one urine drug test during the study period $(\mathrm{n}=98$ ). Prospective 6-month OUD outcomes (treatment retention, substance use recurrence, and buprenorphine continuation) were abstracted from the medical record. Univariate analyses explored differences by gender. Multivariate regressions assessed predictors of OUD outcomes. Participants were 53\% women and middle-aged (47 \pm 12 years). The majority (59\%) had been in treatment for at least 1 year at study enrollment. Substance use recurrence was common, but many individuals remained in treatment. OUD outcomes did not differ by gender. Older age and absence of injection opioid use history were significant predictors of treatment retention and buprenorphine continuation. When provided access to high-quality treatment, Black adults with OUD demonstrate positive outcomes. Addressing structural racism and developing culturally informed treatment interventions are necessary to improve access to high-quality care for this community.
\end{abstract}

Keywords Black adults · Opioid use disorder (OUD) · Medication for opioid use disorder (MOUD) · Buprenorphine · Social determinants of health

\section{Introduction}

Within the USA, Black individuals experience health disparities across a broad range of chronic health conditions, including substance use disorders (SUD) [1]. Notably, structural racism magnifies racial disparities and inequities in social determinants of health that impact SUD morbidity, mortality, and treatment outcomes for Black people [2, 3]. For example, Black individuals are disproportionately burdened with the

Anna Beth Parlier-Ahmad

parlierab@vcu.edu

1 Department of Psychology, Virginia Commonwealth University, 806 W. Franklin St, Richmond, VA 23284, USA

2 Department of Obstetrics and Gynecology and Institute for Drug and Alcohol Studies, Virginia Commonwealth University, 1250 E. Marshall St, Richmond, VA 23298, USA negative consequences associated with substance use including incarceration, violence, stigma, and health problems [4, 5]. Additionally, Black people face systemic barriers to SUD treatment and recovery through discriminatory practices affecting income, education, employment, housing, healthcare, and criminal justice systems [3, 6-8]. Therefore, Black individuals with SUD tend to have lower odds of initiating SUD treatment despite presenting with greater addiction severity and, even after treatment engagement, suffer from worse treatment outcomes such as treatment discontinuation and continued substance use [9-13].

The opioid crisis increased awareness of addiction as a public health concern and improved SUD treatment accessibility within specific subgroups of the population, namely, White, middle-class, suburban, and rural populations. In stark contrast to the national response to the 1980 s cocaine epidemic ("war on drugs") which criminalized substance use, particularly in Black communities, the response to the opioid epidemic has largely portrayed White individuals with opioid 
addiction as "victims" to inappropriate healthcare practices (i.e., overprescribing of prescription opioids) rather than people with moral failings [5]. The devastating impact of the opioid epidemic within Black America has received less attention than other subgroups at least in part due to the harmful stereotypes and biases of addiction in Black communities that have been perpetuated in both medical practice and society at large $[2,5,14]$. Additionally, due to structural racism, Black people are underrepresented in national epidemiological studies, so prevalence of opioid use among Black communities may be underestimated [15].

Opioid use, including both illicit opioid use and prescription opioid misuse, and overdose deaths among Black individuals are continuing to increase at alarmingly high rates and differentially by gender [15-18]. Between 2010 and 2017, Black individuals experienced the largest increase in drugrelated overdose deaths [19]. Additionally, overdose deaths involving synthetic opioids, like fentanyl, are increasing faster among Black individuals than any other racial group [2]. One contributing factor to these devastating disparities in opioidrelated mortality by race is the concurrent pervasive racial disparities in opioid use disorder (OUD) treatment. Black individuals with OUD are less likely than their White counterparts to enter treatment for OUD [20] or receive medication for opioid use disorder (MOUD), which effectively reduces overdose risk and improves health outcomes [21]. Among those receiving treatment for OUD, Black individuals have reduced odds of completing residential treatment [22] or remaining in MOUD treatment for at least 1 year compared to White individuals [23]. Systemic inequities such as lower income with greater reliance upon public insurance sources, lack of transportation and childcare, residential segregation, discrimination, and racial stigma serve as significant barriers impacting treatment access and quality $[2,5$, 24]. However, when Black individuals are provided access to high-quality OUD treatment, they demonstrate significant improvements [25].

MOUD is the gold standard treatment for OUD and is consistently associated with better OUD treatment outcomes including higher treatment retention rates and reduced substance use recurrence compared to non-pharmacological therapies for OUD [26, 27], yet treatment outcomes vary widely by race, age, sex and gender, treatment setting, and polysubstance use [28, 29]. Although predictors of MOUD treatment outcomes have been identified broadly [23, 28, 30-33], little is known about within-group differences in Black adults receiving MOUD, especially for MOUD with buprenorphine. The few studies examining predictors of MOUD outcomes (i.e., substance use and treatment retention) among Black individuals have focused on methadone treatment samples [34, 35]. These studies found polysubstance use and primary injection opioid use negatively impacted shortand long-term outcomes [35], and gender differences emerged in predictors of MOUD outcomes [34]. Given established systems of structural racism and racial disparities that exist along the OUD treatment cascade, additional studies seeking to identify within-group differences may reveal specific psychosocial and clinical strengths that buffer against the systemic issues that disproportionately impact this community.

In line with the recent American Society of Addiction Medicine's statement on racial injustice, future efforts to address health disparities need to prioritize combatting racism and racial inequities in MOUD treatment and employing culturally informed treatment strategies $[2,36]$. One step toward accomplishing this goal is to obtain a better understanding of within-group differences in Black adults receiving MOUD with buprenorphine. The analysis of potential within-group differences may identify specific intervention strategies to alleviate systemic barriers to quality treatment that operate within healthcare settings. Thus, the primary objective of this study is to assess psychosocial and clinical predictors of OUD treatment outcomes through 6 months including (1) treatment retention, (2) substance use recurrence, and (3) buprenorphine continuation among a sample of Black adults receiving buprenorphine for OUD. Based on previous research among broader MOUD treatment populations, we hypothesize that older individuals, women, and those who have a comorbid psychiatric condition, no recent discrimination, stable housing, no history of injection opioid use, and longer treatment durations at the time of the baseline survey will demonstrate better treatment outcomes through the 6-month follow-up period. Although the current study does not analyze racial group differences, it is expected that these predictors operate uniquely within a population of Black individuals with OUD. The secondary objective of this study is to address the NIH mandate to consider sex and gender in research and explore within-group differences in OUD treatment outcomes by gender [37].

\section{Methods}

\section{Design}

This study is a secondary data analysis of a cross-sectional survey study coupled with medical record review. The parent study recruited a convenience sample of English-speaking, adult patients ( $\mathrm{n}=162 ; 97 \%$ response rate) from an outpatient addiction medicine clinic to complete a voluntary, electronic survey between July and September 2019 assessing reproductive and sexual health needs. Patients were recruited through flyers and referrals by clinic staff. Participants who were unable to read had the option to have the survey read aloud by a research assistant in a private space $(n=6)$. Survey completion took an average of $40 \mathrm{~min}$, and participants were compensated $\$ 20$. A medical record abstraction was conducted for 
participants. Virginia Commonwealth University's Institutional Review Board approved the study, and all participants provided verbal consent.

\section{Setting and Participants}

The office-based opioid treatment center provides outpatient addiction services for over 500 adults with approximately $90 \%$ receiving buprenorphine. It is affiliated with a large academic medical center in a Medicaid-expanded state. On-site addiction medicine providers come from multiple specialties, including psychiatry, internal medicine, family medicine, obstetrics and gynecology, and emergency medicine. Most patients are referred from within the academic medical center (e.g., inpatient consults, primary care physicians). At the initial clinic visit, providers complete a comprehensive intake assessment. All patients diagnosed with OUD are offered buprenorphine. Patients typically have follow-up visits at least once every 4 weeks. At follow-up visits, patients undergo urine drug testing; before the COVID-19 pandemic (when this study was conducted), having at least one in-person visit with a drug test every 4 weeks was required to receive continued buprenorphine prescriptions. A comprehensive, recoveryoriented care model is utilized in which patients have access to integrated on-site psychiatric, mental health, case management, and social work services. The clinic prioritizes a lowthreshold, harm reduction approach whenever possible, meaning that established patients with recurrence of substance use are not initially exited from treatment but instead first provided with increased wrap-around support.

For the current secondary data analytic study, participants were limited to those who identified as Black, were receiving MOUD with buprenorphine at the time of the survey, and provided at least one urine drug test during the 28-week follow-up study period $(\mathrm{n}=98,47$ cisgender men, and 52 cisgender women).

\section{Measures}

\section{Opioid Use Disorder Treatment Outcomes}

Treatment outcomes were assessed using substance use treatment record review over seven consecutive 4-week periods (total of 28 weeks) following the survey date. OUD outcomes included the following: (1) treatment retention, (2) substance use recurrence, and (3) buprenorphine continuation.

Treatment retention was operationalized as a dichotomous variable (yes/no) similar to previous studies of OUD treatment outcomes [28] and in line with recommended outcomes specific to MOUD treatment research [38]. The participant's most recent clinic visit and buprenorphine prescription were used to identify the latest 4-week period each patient remained in treatment (range 1-7); if the participant remained in treatment at the seventh 4-week period ( 28 weeks) after survey completion, then the participant was coded was being retained in treatment.

Substance use was operationalized as the percentage of 4week treatment periods (out of seven) with a positive urine drug test for a non-prescribed substance (e.g., opioids, stimulants, cannabis). Buprenorphine continuation was operationalized as the percentage of 4-week treatment periods (out of seven) with a positive urine drug test for buprenorphine. For participants who did not present to treatment during a 4-week period, the urine drug test result for the 4-week period not in treatment was assumed to be positive for non-prescribed substance and negative for buprenorphine because patients in the outpatient study clinic were required to have at least one inperson visit with a urine drug test every 4 weeks in order to receive continued buprenorphine prescriptions. Therefore, if there was not a urine drug test during a 4-week period, the patient would not have received a buprenorphine prescription from their provider. Of all participants, $n=53(54.1 \%)$ had a at least one urine drug test for every 4-week period, and $n=45$ (45.9\%) had at least one 4-week period with an "assumed" positive urine drug test for a non-prescribed substance and negative for buprenorphine. Overall, of all recorded substance use recurrence and buprenorphine continuation, $35 \%$ was "assumed," and $65 \%$ was confirmed by a urine drug test.

\section{Demographic, Psychosocial, and Clinical Variables}

Demographic items included race and gender (cisgender man, cisgender woman, transgender man, transgender woman, other), which were operationalized as social variables, sex assigned at birth (male, female, other), age, employment, education, income, marital status, and living arrangement. Homelessness (yes/no) was defined as living on the street, in a shelter, in a single-room occupancy hotel, or in a car during past 12 months. Recent (i.e., past 12 months) race-based discrimination was assessed via "Have you ever felt you were treated unfairly in getting healthcare services because of your race?" Additionally, substance use-based discrimination was assessed by asking participants "Have you ever felt you were treated unfairly in getting healthcare services because of your drug or alcohol use?" Item responses were dichotomized into yes/no. Two four-item PatientReported Outcomes Measurement Information System (PROMIS) measures assessed current (past 7 days) anxiety and depression symptomatology. Each uses a 5-point frequency scale (1, never, to 5 , always); total scores range from 4 to 20 . For both PROMIS scales, a T-score conversion was used [39]. Norms for the scales are based on US population estimates, with a mean Tscore of $50(\mathrm{SD}=10)$. The Medical Outcomes Study Social Support Survey measured social support using a 6-point frequency scale ( 0 , none of the time, to 5 , all of the time); higher average scores indicate more social support [40]. The number of previous substance use treatment episodes was also assessed. 
Medical and psychiatric comorbidities and insurance status were abstracted from the medical record. Clinical intake assessments were reviewed to obtain substance use history and treatment characteristics. Opioid use items at intake included type of opioid use, route, frequency, age of use onset, and history of overdose (yes/no). Lifetime polysubstance use was defined as any selfreported prior or current use of non-prescribed opioids and nonopioid substances (excluding tobacco). The length of current treatment episode was the number of days between the date of buprenorphine induction and the initial clinic visit date through the survey date. Long-term MOUD treatment with buprenorphine is defined as $\geq 1$ year [23].

\section{Data Analysis}

Descriptive statistics were generated for the overall sample and by gender. Gender stratified OUD outcome comparisons were made using Pearson $\chi 2$ for categorical variables and Ttests for continuous variables. Multivariate logistic and linear regression were used to assess predictors of OUD outcomes including treatment retention, substance use recurrence, and buprenorphine continuation. Variables included in the multivariate models were psychosocial [age, gender, comorbid psychiatric conditions (yes/no), race-based and substance usebased discrimination in a healthcare setting (yes/no), recent homelessness $(\mathrm{y} / \mathrm{n})]$ and clinical [history of injection opioid use (yes/no) and length of current treatment episode]. Participants $(n=8)$ with missing data were excluded from the multivariate analyses. Significance was set at 0.05 , and analyses were performed using SPSS version 26. Due to the small sample size, findings may be underpowered and should be interpreted as preliminary highlighting future areas of study.

\section{Results}

\section{Sample Characteristics}

Demographic, psychosocial, and clinical characteristics are presented in Table 1. Participants $(n=98)$ were middle-aged (47 \pm 12 years). Approximately half were women, unemployed, had public insurance, and earned an annual income of less than $\$ 5,000$. Fewer women were employed than men, and more women than men were single and lived alone with children. Over one-third of all participants reported recent homelessness. Recent discrimination in a healthcare setting was common, with 1 in 4 participants reporting substance use-related discrimination and nearly 1 in 6 reporting racebased discrimination. On average, participants reported social support some to most of the time. Psychiatric and medical comorbidities were common. A higher percentage of women had psychiatric comorbidities than men.
At intake, most participants were using illicit or misusing prescription opioids daily. The most common type of opioid use was heroin; nasal and injection opioid drug use were common (Table 2). On average, participants were $26(\mathrm{SD}=10)$ years of age at the onset of opioid use. Nearly all reported current or past polysubstance use at treatment entry. Men were more likely to have had a previous overdose than women. At the time of the survey, many participants had received long-term MOUD with buprenorphine with the average length of the current treatment episode of $407(\mathrm{SD}=249)$ days (Table 2); men tended to have a shorter treatment duration than women. Importantly, no tests for statistical significance were conducted regarding treatment characteristics, demographic information, and psychosocial functioning; so, values presented in Tables 1 and 2 are not interpretable as significantly significant.

\section{OUD Treatment Outcomes}

Table 3 summarizes OUD treatment outcomes. Most participants $(66 \%)$ remained in treatment through the 28 -week follow-up period. On average, substance use recurrence occurred in $60 \%(\mathrm{SD}=34)$ of 4 -week treatment periods, and buprenorphine was continued in $58 \%$ ( $\mathrm{SD}=$ 27) of 4-week treatment periods. OUD treatment outcomes did not differ significantly by gender regarding treatment retention $(p=.941)$, substance use recurrence $(p=.131)$, and buprenorphine continuation $(p=.401)$. Men had a slightly higher percentage of follow-up periods with substance use recurrence and lower percentage with buprenorphine continuation than women, although these differences were not significantly different.

\section{Predictors of Treatment Outcomes}

Overall, the logistic regression model (Table 4) assessing predictors of treatment retention through the 28 -week period was not statistically significant $[\chi 2(8)=12.9, p=.117$, Nagelkerke $\left.R^{2}=.18\right]$. The multivariate linear regression models (Table 4) did not significantly predict percentage of follow-up periods with substance use recurrence $\left[F(8,81)=1.06, p=.399, R^{2}=\right.$ $.10]$ or buprenorphine continuation $[F(8,81)=1.29, p=.259$, $\left.R^{2}=.11\right]$. Those who were older $(\beta=1.05, p=.039)$ and with no history of injection opioid use $(\beta=.22, p=.006)$ were more likely to remain in treatment when controlling for psychosocial and clinical factors. Additionally, older individuals $(\beta=.61, p=.029)$ and those with no history of injection opioid use $(\beta=-11.86, p=.044)$ were significantly more likely to continue buprenorphine. No variables included in the model were significantly associated with substance use recurrence. Although not significant, those with a psychiatric comorbidity were 2.9 times more 
Table 1 Demographic, psychosocial, and clinical characteristics of Black adults receiving buprenorphine for opioid use disorder

\begin{tabular}{|c|c|c|c|}
\hline Demographic and psychosocial characteristics & $\begin{array}{l}\text { Total } \\
\mathrm{N}(\%) \\
\mathrm{N}=98\end{array}$ & $\begin{array}{l}\text { Men } \\
\mathrm{N}(\%) \\
\mathrm{N}=47\end{array}$ & $\begin{array}{l}\text { Women } \\
\mathrm{N}(\%) \\
\mathrm{N}=52\end{array}$ \\
\hline Age (mean years $\pm \mathrm{SD})$ & $46.9 \pm 12.2$ & $51.2 \pm 10.5$ & $42.9 \pm 12.4$ \\
\hline \multicolumn{4}{|l|}{ Employment } \\
\hline $\begin{array}{l}\text { Employed } \\
\text { Unemployed } \\
\text { Receiving disability }\end{array}$ & $\begin{array}{l}22(22.4) \\
51(52.0) \\
25(25.5)\end{array}$ & $\begin{array}{l}15(31.9) \\
22(46.8) \\
10(21.3)\end{array}$ & $\begin{array}{l}7(13.7) \\
29(56.9) \\
15(29.4)\end{array}$ \\
\hline \multicolumn{4}{|l|}{ Insurance } \\
\hline $\begin{array}{l}\text { Public } \\
\text { Private } \\
\text { None }\end{array}$ & $\begin{array}{l}57(58.2) \\
13(13.3) \\
28(28.6)\end{array}$ & $\begin{array}{l}29(61.7) \\
6(12.8) \\
12(25.5)\end{array}$ & $\begin{array}{l}28(54.9) \\
7(13.7) \\
16(31.4)\end{array}$ \\
\hline \multicolumn{4}{|l|}{ Annual household income } \\
\hline $\begin{array}{l}<\$ 5,000 \\
\$ 5,000 \text { to } 10,000 \\
>\$ 10,000 \\
\text { Not reported }\end{array}$ & $\begin{array}{l}52(53.1) \\
18(18.4) \\
25(25.5) \\
3(3.1)\end{array}$ & $\begin{array}{l}20(42.6) \\
10(21.3) \\
16(34.0) \\
1(2.1)\end{array}$ & $\begin{array}{l}32(62.7) \\
8(15.7) \\
9(17.6) \\
2(3.9)\end{array}$ \\
\hline \multicolumn{4}{|l|}{ Education } \\
\hline $\begin{array}{l}\text { « High school education } \\
\text { High school education } \\
>\text { High school education }\end{array}$ & $\begin{array}{l}26(26.5) \\
45(45.9) \\
27(27.6)\end{array}$ & $\begin{array}{l}14(29.8) \\
23(48.9) \\
10(21.3)\end{array}$ & $\begin{array}{l}12(23.5) \\
22(43.1) \\
17(33.3)\end{array}$ \\
\hline Single marital status & $66(67.3)$ & $28(59.6)$ & $38(74.5)$ \\
\hline $\begin{array}{l}\text { Current living arrangement } \\
\text { With sexual partner } \\
\text { Alone with children } \\
\text { With family/friends } \\
\text { Alone } \\
\text { Other } \\
\text { Not reported }\end{array}$ & $\begin{array}{l}22(22.4) \\
12(12.2) \\
37(37.8) \\
15(15.3) \\
11(11.2) \\
1(1.0)\end{array}$ & $\begin{array}{l}13(27.7) \\
2(4.3) \\
16(34.0) \\
11(23.4) \\
5(10.6) \\
0\end{array}$ & $\begin{array}{l}9(17.6) \\
10(19.6) \\
21(41.2) \\
4(7.8) \\
6(11.8) \\
1(2.0)\end{array}$ \\
\hline Homeless (past 12 months) & $36(37.5)$ & $18(38.3)$ & $18(36.7)$ \\
\hline Substance use discrimination in healthcare setting (past 12 months) & $25(25.5)$ & $11(23.4)$ & $14(27.5)$ \\
\hline Race-based discrimination in healthcare setting (past 12 months) & $15(15.3)$ & $7(14.9)$ & $8(15.7)$ \\
\hline${ }^{\mathrm{a}}$ Social support [median (IQR)] & $3.8(2.8-4.4)$ & $3.4(2.5-4.4)$ & $3.8(2.9-4.4)$ \\
\hline \multicolumn{4}{|l|}{${ }^{\mathrm{b}} \mathrm{PROMIS}($ mean $\pm \mathrm{SD}$ ) } \\
\hline $\begin{array}{l}\text { Depression } \\
\text { Anxiety }\end{array}$ & $\begin{array}{l}54.9 \pm 10.4 \\
56.5 \pm 12.3\end{array}$ & $\begin{array}{l}55.2 \pm 9.7 \\
57.3 \pm 11.9\end{array}$ & $\begin{array}{l}54.6 \pm 11.1 \\
55.8 \pm 12.7\end{array}$ \\
\hline Psychiatric comorbidity & $66(67.3)$ & $28(59.6)$ & $41(87.2)$ \\
\hline Chronic medical comorbidity & $81(82.7)$ & $38(74.5)$ & $40(78.4)$ \\
\hline
\end{tabular}

Note: $S D$, standard deviation; $I Q R$, interquartile range

${ }^{a}$ Social support scale range $0-5$

${ }^{\mathrm{b}}$ PROMIS scales used a T-score conversion (range 0-100, mean T-score $50 \pm 10$ )

likely than those without to remain in treatment through the follow-up period ( $b=1.05, p=.055)$. Substance use-related discrimination was negatively correlated with treatment retention $(b=-.40, p=.498)$ and buprenorphine continuation $(\beta=-6.91, p=.304)$, while race-based discrimination was negatively correlated with treatment retention $(b=-.15, p=.823)$ and positively correlated with substance use recurrence $(\beta=5.56, p=$ .626 ); however, none of these correlations reached the level of significance (Table 4).

\section{Discussion}

The purpose of the current study was to identify psychosocial and clinical strengths that buffer against the systemic issues that disproportionately impact Black communities who seek MOUD treatment. Findings of the current study add to the literature by providing a general snapshot of Black patients engaged in MOUD treatment with buprenorphine and highlighting the resiliency of this community. From a strength-based perspective, study findings showed that despite 
Table 2 Substance use history and treatment characteristics of Black adults receiving buprenorphine for opioid use disorder

\begin{tabular}{llll}
\hline Substance use history and treatment characteristics & Total & Men & Women \\
& $\mathrm{N}(\%)$ & $\mathrm{N}(\%)$ & $\mathrm{N}(\%)$ \\
& $\mathrm{N}=98$ & $\mathrm{~N}=47$ & $\mathrm{~N}=52$ \\
\hline Type of opioid use & & & \\
Heroin only & $72(73.5)$ & $41(87.2)$ & $31(60.8)$ \\
Prescription opioid only & $8(8.2)$ & $1(2.1)$ & $7(13.7)$ \\
Both & $18(18.4)$ & $5(10.6)$ & $13(25.5)$ \\
Most serious route of opioid use & $42(42.9)$ & $23(48.9)$ & $19(37.3)$ \\
Injection & $40(40.8)$ & $19(40.4)$ & $21(41.2)$ \\
Nasal & $8(8.2)$ & $1(2.1)$ & $7(13.7)$ \\
Oral & $8(8.2)$ & $4(8.5)$ & $4(7.8)$ \\
Not reported & & & \\
Daily opioid use at treatment entry (nonprescription use) & $81(82.7)$ & $40(85.1)$ & $41(80.4)$ \\
Age onset opioid use (mean \pm SD) & $26.0 \pm 10.4$ & $25.4 \pm 11.0$ & $26.6 \pm 10.0$ \\
History overdose & $22(22.4)$ & $13(27.7)$ & $9(17.6)$ \\
Lifetime polysubstance use & $92(93.9)$ & $46(97.9)$ & $46(90.2)$ \\
Prior self-help group (AA/NA) & $11(11.2)$ & $6(12.8)$ & $5(9.8)$ \\
${ }^{a}$ Prior specialty SUD treatment & $70(71.4)$ & $35(74.5)$ & $35(68.6)$ \\
${ }^{b}$ Long-term MOUD with buprenorphine $(\geq 1$ year) & $58(59.2)$ & $25(53.2)$ & $33(64.7)$ \\
${ }^{b}$ Length of current treatment episode (Mean \pm SD; days) & $406.9 \pm 249.4$ & $358.6 \pm 247.0$ & $451.4 \pm 245.6$ \\
\hline & & & \\
\hline
\end{tabular}

Notes: $S D$, standard deviation; $A A$, alcoholics anonymous; $N A$, narcotics anonymous

${ }^{\text {a }}$ Prior specialty SUD treatment includes inpatient/residential, intensive outpatient, medication for OUD, and behavioral health services for SUD

${ }^{\mathrm{b}}$ At time of baseline survey completion some having increased psychosocial vulnerabilities such as race-based discrimination, recent homelessness, and presence of a psychiatric comorbidity, participants achieved OUD treatment outcomes similar to other populations receiving MOUD with buprenorphine (e.g., $57 \%$ substance use recurrence at 24 weeks [41]; 57\% 6-month retention [28]). Additionally, many individuals engaged in MOUD treatment remained in treatment throughout the duration of the study follow-up period, despite observed substance use recurrence. Previous findings among a sample of Black individuals with OUD have shown that when provided access to high-quality MOUD treatment with buprenorphine, they experienced improved quality of life and were empowered to reduce substance use and risk behaviors [25]. As such, engagement in MOUD treatment serves as a buffer to several previously established psychosocial factors that have been shown to negatively impact OUD treatment outcomes.

Consistent with previous literature, older age and absence of injection opioid use history were significant predictors of positive OUD outcomes when controlling for psychosocial and clinical factors. Previous studies among diverse samples of adults in MOUD treatment have found that younger individuals were at higher risk of poor retention rates [23, 28, 30, 31]. Similarly, our findings suggest that older individuals not only have better retention rates but are also more likely to continue buprenorphine [42]. Because addiction is a chronic

Table 3 Opioid use disorder treatment outcomes among Black adults receiving buprenorphine for opioid use disorder

\begin{tabular}{|c|c|c|c|c|}
\hline Opioid use disorder treatment outcomes & $\begin{array}{l}\text { Total } \\
\mathrm{N}=98\end{array}$ & $\begin{array}{l}\text { Men } \\
\mathrm{N}=47\end{array}$ & $\begin{array}{l}\text { Women } \\
\mathrm{N}=52\end{array}$ & $P$ value \\
\hline Treatment retention: remained in treatment for 7 treatment periods $[\mathrm{N}(\%)]$ & $65(66.3)$ & $31(66.0)$ & $34(66.7)$ & .941 \\
\hline $\begin{array}{l}\text { Substance use recurrence: percent of treatment periods with } \\
\text { urine testing positive for non-prescribed substance out of } 7 \text { (mean } \pm \text { SD) }\end{array}$ & $60 \pm 34.2$ & $65.7 \pm 33.8$ & $55.2 \pm 34.2$ & .131 \\
\hline $\begin{array}{l}\text { Buprenorphine continuation: percent of treatment periods with urine } \\
\text { testing positive for buprenorphine out of } 7(\text { mean } \pm S D)\end{array}$ & $58.2 \pm 25.6$ & $55.9 \pm 25.6$ & $60.2 \pm 25.6$ & .409 \\
\hline
\end{tabular}

Notes: $S D$, standard deviation 
Table 4 Predictors of opioid use disorder treatment outcomes among Black adults receiving buprenorphine for opioid use disorder $(\mathrm{n}=90)$

\begin{tabular}{llll}
\hline Treatment retention (y/n) & AOR $(95 \% \mathrm{CI})$ & $\mathrm{b}$ & P value \\
\hline Age & $1.05(1.00,1.10)$ & .05 & $.039^{*}$ \\
Women & $1.06(.37,3.05)$ & .06 & .887 \\
Mental health comorbidity & $2.90(.97,8.49)$ & 1.05 & .055 \\
Recent race-based discrimination & $.86(.21,3.50)$ & -.15 & .823 \\
Recent substance use discrimination & $.67(.22,2.08)$ & -.40 & .498 \\
Recent homelessness & $.92(.33,2.58)$ & -.08 & .885 \\
History of injection opioid use & $.22(.08, .65)$ & -1.50 & $.006^{*}$ \\
Length of current treatment episode & $1.00(.99,1.00)$ & .00 & .879 \\
Substance use recurrence (out of 7 periods) & $\mathrm{Beta}(95 \% \mathrm{CI})$ & $\mathrm{b}$ & $\mathrm{P}$ value \\
Age & $-.25(-.96, .46)$ & -.09 & .488 \\
Women & $-8.03(-24.40,8.35)$ & -.12 & .332 \\
Mental health comorbidity & $-6.49(-23.15,10.18)$ & -.09 & .441 \\
Recent race-based discrimination & $5.56(-17.03,28.15)$ & .06 & .626 \\
Recent substance use discrimination & $-4.88(-22.65,12.88)$ & -.06 & .586 \\
Recent homelessness & $4.88(-10.92,20.69)$ & .07 & .540 \\
History of injection opioid use & $10.87(-4.57,26.31)$ & .16 & .165 \\
Length of current treatment episode & $-.026(-.06, .01)$ & -.19 & .101 \\
Buprenorphine continuation (of 7 periods) & Beta $(95 \% \mathrm{CI})$ & $\mathrm{b}$ & P value \\
Age & $.61(.08,1.14)$ & .29 & $.026^{*}$ \\
Women & $7.28(-4.97,19.52)$ & .14 & .241 \\
Mental health comorbidity & $6.64(-5.83,19.11)$ & .12 & .292 \\
Recent race-based discrimination & $3.58(-13.32,20.48)$ & .05 & .674 \\
Recent substance use discrimination & $-6.91(-20.19,6.38)$ & -.12 & .304 \\
Recent homelessness & $1.69(-10.13,13.52)$ & .03 & .776 \\
History of injection opioid use & $-11.86(-23.41,-.30)$ & -.23 & $.044^{*}$ \\
Length of current treatment episode & $.01(-.02, .03)$ & .06 & .611 \\
\hline & & & \\
\hline
\end{tabular}

*Indicates significant at $\mathrm{p} \leq .05$ medical disease, older individuals likely have more experience coping with addiction and may have a better understanding of their disease as well as the treatment that is most beneficial for them. Future research investigating ways to more actively engage young people in MOUD treatment is warranted [31]. Additionally, we found that individuals with an injection opioid use history were less likely to remain in treatment and continue buprenorphine. Previous research among Black patients receiving methadone for OUD demonstrated that primary injection opioid use negatively impacted substance use but not treatment retention [35]. This heterogeneity across studies may reflect how injection drug use commonly occurs in context with greater psychosocial vulnerabilities, medical complications, and SUD severity, which have been broadly shown across racially/ethnically heterogeneous populations with OUD [43, 44]. Approximately 2 in 5 study participants reported a history of injection opioid use. These individuals may benefit from additional support from comprehensive wrap-around services (e.g., peer recovery support) to improve buprenorphine treatment engagement and retention [45].
Although no other psychosocial or clinical factors examined significantly predicted OUD treatment outcomes among this sample, those with a psychiatric comorbidity were nearly 3 times more likely to remain in treatment through the study period than those without a psychiatric comorbidity. Prior work has identified a relationship between the presence of a psychiatric comorbidity and improved treatment retention as well as reduced substance use, among other populations of individuals with OUDs [23, 32]. Psychiatric comorbidities may increase access to SUD treatment through mental health providers [46] and prompt individuals to seek and engage in SUD treatment [47]. The potential antidepressant effects of buprenorphine may also contribute to longer treatment retention as the medication may improve depressive symptoms as well as cravings and withdrawal symptoms [48]. However, more research is needed to understand the mechanism behind the relationship between the presence of a psychiatric comorbidity and improved treatment retention. Additionally, more strength-based factors such as coping skills, sense of cultural identity, and spirituality need to be further explored as potential predictors of MOUD treatment success, so 
inventions can be tailored to maximize individual strengths and improve treatment outcomes $[49,50]$.

Among our sample of Black adults receiving buprenorphine for OUD, no significant gender differences emerged across OUD treatment outcomes. However, compared to men, women did have fewer treatment periods with a positive urine drug test and more treatment periods with buprenorphine continuation. Literature has shown, compared to men, women typically experience more psychosocial and medical vulnerabilities that hinder OUD treatment initiation and engagement $[51,52]$. These treatment barriers are even greater for Black women who face additional systemic barriers [53]. Even with the additional barriers to treatment, women tend to have similar treatment outcomes as men once engaged in OUD treatment [34, 54]. Recent studies have even shown a possible female advantage with buprenorphine in OUD outcomes that has not been identified with previous forms of OUD treatment [55]. More research with larger samples is needed to better understand the potential gender differences in OUD outcomes among Black individuals in MOUD treatment with buprenorphine and advance gender-informed interventions.

\section{Clinical Implications and Recommendations}

Among our sample, substance use-related and race-based discrimination in a healthcare setting were not uncommon, and both were negatively correlated with OUD outcomes. Research has shown that substance use stigma toward individuals with OUD is prevalent within the healthcare community [56] and has been associated with a negative perception of treatment helpfulness and ultimately SUD treatment discontinuation among Black people [57]. Thus, healthcare providers need to be involved with OUD education efforts and substance use stigma-reduction strategies [58]. Additionally, racial discrimination is a source of chronic psychological distress and pain which has been associated with substance use and negative outcomes among Black individuals [49]. Although structural racism is the root cause of racial health disparities, racial discrimination by clinicians reinforces these systems and contributes to poor health outcomes among Black people [3]. Ongoing training on anti-racism, implicit bias, microaggressions, and shared decision-making is needed for healthcare professionals $[59,60]$. Further, it is vitally important for healthcare systems, clinicians, and researchers to foster environments that embrace cultural humility and empower Black patients [60]. Addressing intersectional anti-Black and substance use stigma and discrimination in healthcare may help reduce barriers, improve overall health, and support recovery of Black people with OUD.

While we still have room for improvement in addressing structural racism within our SUD treatment program, we hypothesize that several unique program characteristics helped improve access and quality of care within this community and, in turn, improve outcomes. First, the clinic prioritizes a lowthreshold, harm reduction approach and utilizes a recoveryoriented care model. This means that established patients with recurrence of substance use are not initially exited from treatment. Instead, patients are supported by increased wraparound services including integrated psychiatry, counseling, and case management. Patients who need a higher level of care are provided a warm handoff to a community-based program. This approach aligns with previous studies that have shown improvement in OUD outcomes with harm reduction programs $[61,62]$. Second, the SUD treatment clinic has onsite addiction medicine providers from multiple specialties including psychiatry, internal medicine, family medicine, obstetrics and gynecology, and emergency medicine who are waivered to prescribe buprenorphine. Thus, patients have access to integrated addiction treatment and medical care which has also been shown to improve outcomes [63]. Lastly, our SUD treatment program is affiliated with a large academic medical center that serves as a safety net for the region. Most patients are referred to the SUD treatment program by providers within the academic medical center (e.g., inpatient consults, primary care physicians) which likely increases access to and facilitates initiation of SUD treatment services. At a program level, these policies and characteristics help combat the systemic barriers that disproportionately impact this community. We need to move toward a system in which adoption of such policies within SUD treatment settings is the norm rather than the exception.

\section{Limitations}

The current study findings should be considered within the context of several limitations and thus directions for future research. First and foremost, given our small sample size, our study may be underpowered impacting the ability to detect statistically significant differences. Because of this limitation, findings should be interpreted as preliminary; future adequately powered, hypothesis-driven studies are needed to further clarify predictors of MOUD outcomes within this unique population. Participants self-selected into this convenience sample from one outpatient clinic, and this self-selection may have contributed to positive retention rates among the sample. Findings may not be representative of those who chose not to participate in the study and may not generalize to individuals who are engaged with alternative treatment modalities, who do not remain engaged in treatment, or who are nontreatment seeking. As such, future research should consider recruiting a broader and larger sample to address these generalizability concerns. Social desirability and information bias may have skewed our results as participants were asked to self-report on several sensitive issues in the survey, and identification of variables obtained from our chart review relied on 
provider documentation. Given the outpatient setting of the study site, we were unable to confirm all reported substance use recurrence and buprenorphine continuation with a urine drug test. Based on study site clinical procedures, presented results include 35\% "assumed" outcomes (i.e., positive urine drug test for a non-prescribed substance and negative for buprenorphine) and, thus, may be an overestimation of substance use recurrence and underestimation of buprenorphine continuation. Gender identity was assessed in the survey, but no participants identified as a gender minority. Thus, our analyses were limited to a cisgender conceptualization of gender identity. Future research can build upon the current study by incorporating self-reported outcome measures of psychosocial functioning and holistic recovery in addition to the traditional OUD treatment outcomes to better understand the experience of Black communities in MOUD treatment. Despite these limitations, to our knowledge, this study is the first to describe within-group differences between Black men and women engaged in outpatient MOUD with buprenorphine lending our findings to be directly applicable to clinical care.

\section{Conclusions}

When Black adults with OUD are provided access to highquality MOUD treatment, they demonstrate positive OUD outcomes. Thus, we need to improve and ensure access to high-quality care for this community. The COVID-19 pandemic has exacerbated systemic racial inequities and in turn the racial disparities in SUD morbidity, mortality, and treatment outcomes [64]. It is imminent that we take steps now to appropriately address structural racism and develop culturally informed SUD treatment interventions. The current study provides data-driven clinical recommendations for how to begin to do so.

\begin{abstract}
Author Contribution All authors contributed to the study conception and design. Material preparation, data collection, and analysis were performed by Caitlin E. Martin and Anna Beth Parlier-Ahmad. The first draft of the manuscript was written by Anna Beth Parlier-Ahmad. Caitlin E. Martin and Mickeal Pugh Jr. reviewed and edited previous versions of the manuscript. All authors read and approved the final manuscript.
\end{abstract}

Funding Anna Beth Parlier-Ahmad reports receiving NIDA T32DA007027 award (PI, Dr. William Dewey). Dr. Martin is supported by CTSA award No. UL1TR002649 and No. KL2TR002648 from the National Center for Advancing Translational Sciences. This study was supported by the Jeanann Dunlap Foundation. Its contents are solely the responsibility of the authors and do not necessarily represent official views of the National Center for Advancing Translational Sciences or the National Institute on Drug Abuse. Funders were not directly involved in the preparation of this research manuscript.

Data Availability Data are available upon request.

\section{Declarations}

Ethics Approval The Institutional Review Board of Virginia Commonwealth University reviewed and approved all study procedures.

Consent to Participate Informed consent was obtained from all participants included in the study.

Consent for Publication Not applicable.

Conflict of Interest The authors declare no competing interests.

\section{References}

1. National Center for Health S. Health, United States. Health, United States, 2015: with special feature on racial and ethnic health disparities. Hyattsville (MD): National Center for Health Statistics (US); 2016.

2. SAMHSA. The opioid crisis and the Black/African American population: an urgent issue 2020. Available from: https://store.samhsa. gov/product/The-Opioid-Crisis-and-the-Black-African-AmericanPopulation-An-Urgent-Issue/PEP20-05-02-001.

3. Bailey ZD, Krieger N, Agénor M, Graves J, Linos N, Bassett MT. Structural racism and health inequities in the USA: evidence and interventions. Lancet. 2017;389(10077):1453-63. https://doi.org/ 10.1016/s0140-6736(17)30569-x.

4. Matsuzaka S, Knapp M. Anti-racism and substance use treatment: addiction does not discriminate, but do we? J Ethn Subst Abus. 2019:1-27. Epub 2019/01/16. https://doi.org/10.1080/15332640. 2018.1548323.

5. Santoro TN, Santoro JD. Racial bias in the US opioid epidemic: a review of the history of systemic bias and implications for care. Cureus. 2018;10(12):e3733. Epub 2019/02/26. https://doi.org/10. 7759 /cureus.3733.

6. Verissimo ADO, Grella CE. Influence of gender and race/ethnicity on perceived barriers to help-seeking for alcohol or drug problems. J Subst Abus Treat. 2017;75:54-61. https://doi.org/10.1016/j.jsat. 2016.12.013.

7. Pinedo M, Zemore S, Mulia N. Black-White differences in barriers to specialty alcohol and drug treatment: findings from a qualitative study. J Ethn Subst Abus. 2020:1-15. Epub 2020/01/22. https://doi. org/10.1080/15332640.2020.1713954.

8. Saloner B, Le Cook B. Blacks and Hispanics are less likely than whites to complete addiction treatment, largely due to socioeconomic factors. Health Aff (Millwood). 2013;32(1):135-45. Epub 2013/01/09. https://doi.org/10.1377/hlthaff.2011.0983.

9. Schmidt LA, Ye Y, Greenfield TK, Bond J. Ethnic disparities in clinical severity and services for alcohol problems: results from the National Alcohol Survey. Alcohol Clin Exp Res. 2007;31(1):4856. Epub 2007/01/09. https://doi.org/10.1111/j.1530-0277.2006. 00263.x.

10. Pinedo M. A current re-examination of racial/ethnic disparities in the use of substance abuse treatment: do disparities persist? Drug Alcohol Depend. 2019;202:162-7. Epub 2019/07/29. https://doi. org/10.1016/j.drugalcdep.2019.05.017.

11. Lewis B, Hoffman L, Garcia CC, Nixon SJ. Race and socioeconomic status in substance use progression and treatment entry. $\mathrm{J}$ Ethn Subst Abus. 2018;17(2):150-66. Epub 2017/08/29. https:// doi.org/10.1080/15332640.2017.1336959.

12. Mennis J, Stahler GJ. Racial and ethnic disparities in outpatient substance use disorder treatment episode completion for different 
substances. J Subst Abus Treat. 2016;63:25-33. Epub 2016/01/29. https://doi.org/10.1016/j.jsat.2015.12.007.

13. Sahker E, Pro G, Sakata M, Furukawa TA. Substance use improvement depends on race/ethnicity: outpatient treatment disparities observed in a large US national sample. Drug Alcohol Depend. 2020;213:108087. Epub 2020/06/04. https://doi.org/10.1016/j. drugalcdep.2020.108087.

14. Drake J, Charles C, Bourgeois JW, Daniel ES, Kwende M. Exploring the impact of the opioid epidemic in Black and Hispanic communities in the United States. Drug Sci Policy Law. 2020;6. https://doi.org/10.1177/2050324520940428.

15. Jordan A, Mathis M, Haeny A, Funaro M, Paltin D, Ransome Y. An Evaluation of opioid use in Black communities: a rapid review of the literature. Harv Rev Psychiatry. 2021;29(2):108-30. Epub 2021/03/06. https://doi.org/10.1097/hrp.0000000000000285.

16. Lippold KM. Jones, C. M., Olsen, E. O., Giroir, B. P. Racial/ethnic and age differences in opioid involved overdose deaths in adults US 2015-2017. Morb Mortal Wkly Rep. 2019;63(43):967-73.

17. Furr-Holden D, Milam AJ, Wang L, Sadler R. African Americans now outpace whites in opioid-involved overdose deaths: a comparison of temporal trends from 1999 to 2018. Addiction. 2021;116(3): 677-83. Epub 2020/08/28. https://doi.org/10.1111/add.15233.

18. Nicholson HL, Ford JA. Correlates of prescription opioid misuse among Black adults: findings from the 2015 National Survey on Drug Use and Health. Drug Alcohol Depend. 2018;186:264-7. Epub 2018/02/27. https://doi.org/10.1016/j.drugalcdep.2017.12. 006.

19. Woolf SH, Schoomaker H. Life expectancy and mortality rates in the United States, 1959-2017. Jama. 2019;322(20):1996-2016. Epub 2019/11/27. https://doi.org/10.1001/jama.2019.16932.

20. Wu LT, Zhu H, Swartz MS. Treatment utilization among persons with opioid use disorder in the United States. Drug Alcohol Depend. 2016;169:117-27. Epub 2016/11/05. https://doi.org/10. 1016/j.drugalcdep.2016.10.015.

21. SAMHSA. Medications for opioid use disorder. Treatment Improvement Protocol (TIP) Series 63. Publication No. PEP2002-01-006. Rockville: Substance Abuse and Mental Health Services Administration; 2020.

22. Stahler GJ, Mennis J. Treatment outcome disparities for opioid users: are there racial and ethnic differences in treatment completion across large US metropolitan areas? Drug Alcohol Depend. 2018;190:170-8. Epub 2018/07/25. https://doi.org/10.1016/j. drugalcdep.2018.06.006.

23. Weinstein ZM, Kim HW, Cheng DM, Quinn E, Hui D, Labelle CT, et al. Long-term retention in office based opioid treatment with buprenorphine. J Subst Abus Treat. 2017;74:65-70. Epub 2017/01/31. https://doi.org/10.1016/j.jsat.2016.12.010.

24. Schiff DM, Nielsen T, Hoeppner BB, Terplan M, Hansen H, Bernson D, et al. Assessment of racial and ethnic disparities in the use of medication to treat opioid use disorder among pregnant women in Massachusetts. JAMA Network Open. 2020;3(5): e205734. Epub 2020/05/27. https://doi.org/10.1001/ jamanetworkopen.2020.5734. PubMed PMID: 32453384; PubMed Central PMCID: PMCPMC7251447 Centers for Disease Control and Prevention during the conduct of the study. Dr Krans reported receiving grants from Gilead Sciences, Merck, and the National Institutes of Health outside the submitted work. Dr Wilens reported receiving grants from the National Institute on Drug Abuse, National Institutes of Health; other from Cambridge University Press, Guilford Press, Ironshore, KemPharm, Otsuka Pharmaceutical, and Vallon Pharmaceuticals; personal fees from Bay Cove Human Services, the Gavin Foundation, US Major League Baseball, US Minor League Baseball, and the US National Football League outside the submitted work; and receiving royalties as a co-owner (with Ironshore) of the patent for the Before School Functioning Questionnaire, a copyrighted diagnostic questionnaire. No other disclosures were reported.

25. Mitchell SG, Gryczynski J, Schwartz RP, O ' Grady KE, Olsen YK, Jaffe JH. A randomized trial of intensive outpatient (IOP) vs. standard outpatient (OP) buprenorphine treatment for African Americans. Drug Alcohol Depend. 2013;128(3):222-9. Epub 2012/09/25. https://doi.org/10.1016/j.drugalcdep.2012.08.027.

26. Timko C, Schultz NR, Cucciare MA, Vittorio L, Garrison-Diehn C. Retention in medication-assisted treatment for opiate dependence: a systematic review. J Addict Dis. 2015;35(1):22-35. https://doi.org/ 10.1080/10550887.2016.1100960.

27. Wakeman SE, Larochelle MR, Ameli O, Chaisson CE, McPheeters JT, Crown WH, et al. Comparative effectiveness of different treatment pathways for opioid use disorder. JAMA Netw Open. 2020;3(2):e1920622-e. https://doi.org/10.1001/jamanetworkopen. 2019.20622.

28. O ' Connor AM, Cousins G, Durand L, Barry J, Boland F. Retention of patients in opioid substitution treatment: a systematic review. PLoS One. 2020;15(5):e0232086. Epub 2020/05/15. https://doi.org/10.1371/journal.pone.0232086.

29. Gryczynski J, Mitchell SG, Jaffe JH, Kelly SM, Myers CP, O Grady KE, et al. Retention in methadone and buprenorphine treatment among African Americans. J Subst Abus Treat. 2013;45(3): 287-92. Epub 2013/04/10. https://doi.org/10.1016/j.jsat.2013.02. 008.

30. Lee CS, Liebschutz JM, Anderson BJ, Stein MD. Hospitalized opioid-dependent patients: exploring predictors of buprenorphine treatment entry and retention after discharge. Am J Addict. 2017;26(7):667-72. Epub 2017/03/23. https://doi.org/10.1111/ ajad.12533.

31. Damian AJ, Mendelson T, Agus D. Predictors of buprenorphine treatment success of opioid dependence in two Baltimore City grassroots recovery programs. Addict Behav. 2017;73:129-32. Epub 2017/05/16. https://doi.org/10.1016/j.addbeh.2017.05.010.

32. Peckham AD, Griffin ML, McHugh RK, Weiss RD. Depression history as a predictor of outcomes during buprenorphine-naloxone treatment of prescription opioid use disorder. Drug Alcohol Depend. 2020;213:108122. Epub 2020/06/22. https://doi.org/10. 1016/j.drugalcdep.2020.108122.

33. Justesen K, Ah S, Sherman MD, Lonergan-Cullum M, Nissly T, Levy R. Predictors of family medicine patient retention in opioid medication-assisted treatment. J Am Board Fam Med. 2020;33(6): 848-57. Epub 2020/11/22. https://doi.org/10.3122/jabfm.2020.06. 200086.

34. Lister JJ, Brown S, Greenwald MK, Ledgerwood DM. Genderspecific predictors of methadone treatment outcomes among African Americans at an urban clinic. Subst Abus. 2019;40(2): 185-93. Epub 2019/03/20. https://doi.org/10.1080/08897077. 2018.1547810 .

35. Lister JJ, Greenwald MK, Ledgerwood DM. Baseline risk factors for drug use among African-American patients during first-month induction/stabilization on methadone. J Subst Abus Treat. 2017;78: 15-21. Epub 2017/05/31. https://doi.org/10.1016/j.jsat.2017.04. 007.

36. ASAM. ASAM's commitment to addressing racial injustice and health disparities: American Society of Addiction Medicine; 2020 [updated 12 June 2020; cited 202030 June]. Available from: https://www.asam.org/Quality-Science/publications/magazine/ $\mathrm{read} /$ article/2020/06/12/asam-s-commitment-to-addressing-racialinjustice-and-health-disparities.

37. Wetherington CL. Sex-gender differences in drug abuse: a shift in the burden of proof? Exp Clin Psychopharmacol. 2007;15(5):4117. https://doi.org/10.1037/1064-1297.15.5.411.

38. Williams AR, Nunes EV, Bisaga A, Pincus HA, Johnson KA, Campbell AN, et al. Developing an opioid use disorder treatment cascade: a review of quality measures. J Subst Abus Treat. 2018;91: 
57-68. Epub 2018/06/19. https://doi.org/10.1016/j.jsat.2018.06. 001.

39. Measures H. PROMIS scoring manuals 2020 [9/21/20]. Available from: https://www.healthmeasures.net/index.php?option=com content $\&$ view $=$ article $\&$ id= $=180 I \&$ temid $=994$.

40. Sherbourne CD, Stewart AL. The MOS social support survey. Soc Sci Med (1982). 1991;32(6):705-14 Epub 1991/01/01.

41. Lee JD, Nunes EV, Novo P, Bachrach K, Bailey GL, Bhatt S, et al. Comparative effectiveness of extended-release naltrexone versus buprenorphine-naloxone for opioid relapse prevention (X:BOT): a multicentre, open-label, randomised controlled trial. Lancet. 2018;391(10118):309-18. https://doi.org/10.1016/s0140-6736(17) 32812-x.

42. McLellan AT, Lewis DC, O ' Brien CP, Kleber HD. Drug dependence, a chronic medical illness: implications for treatment, insurance, and outcomes evaluation. Jama. 2000;284(13):1689-95. Epub 2000/10/04. https://doi.org/10.1001/jama.284.13.1689.

43. Darke S, Hetherington K, Ross J, Lynskey M, Teesson M. Noninjecting routes of administration among entrants to three treatment modalities for heroin dependence. Drug Alcohol Rev. 2004;23(2): 177-83. Epub 2004/09/17. https://doi.org/10.1080/ 095952304100017044163.

44. Fischer B, Manzoni P, Rehm J. Comparing injecting and noninjecting illicit opioid users in a multisite Canadian sample (OPICAN Cohort). Eur Addict Res. 2006;12(4):230-9. Epub 2006/09/14. https://doi.org/10.1159/000094425.

45. NIDA. Principles of drug addiction treatment: a research-based guide (third edition): behavioral therapies 2018 [cited 2020 November 29]. Available from: https://www.drugabuse.gov/ publications/principles-drug-addiction-treatment-research-basedguide-third-edition/evidence-based-approaches-to-drug-addictiontreatment/behavioral-therapies.

46. Chen LY, Strain EC, Crum RM, Mojtabai R. Gender differences in substance abuse treatment and barriers to care among persons with substance use disorders with and without comorbid major depression. J Addict Med. 2013;7(5):325-34. Epub 2013/10/05. https:// doi.org/10.1097/ADM.0b013e31829b7afe.

47. Harris KMaE, M. J. Use of mental health care and SU treatment among adults with co-occurring disorders. Psychiatr Serv. 2005;56(8):954-9.

48. Saxena PP, Bodkin JA. Opioidergic agents as antidepressants: rationale and promise. CNS Drugs. 2019;33(1):9-16. Epub 2018/11/ 16. https://doi.org/10.1007/s40263-018-0584-7.

49. Stevens-Watkins D, Perry B, Harp KL, Oser CB. Racism and illicit drug use among African American women: the protective effects of ethnic identity, affirmation, and behavior. J Black Psychol. 2012;38(4):471-96. Epub 2012/11/01. https://doi.org/10.1177/ 0095798412438395

50. Heinz A, Epstein DH, Preston KL. Spiritual/religious experiences and in-treatment outcome in an inner-city program for heroin and cocaine dependence. J Psychoactive Drugs. 2007;39(1):41-9. Epub 2007/05/26. https://doi.org/10.1080/02791072.2007.10399863.

51. Campbell ANC, Barbosa-Leiker C, Hatch-Maillette M, Mennenga SE, Pavlicova M, Scodes J, et al. Gender differences in demographic and clinical characteristics of patients with opioid use disorder entering a comparative effectiveness medication trial. Am J Addict. 2018;27(6):465-70. Epub 2018/08/15. https://doi.org/10.1111/ ajad. 12784.
52. Polak K, Haug NA, Drachenberg HE, Svikis DS. Gender considerations in addiction: implications for treatment. Curr Treat Options Psychiatry. 2015;2(3):326-38 Epub 2015/09/29.

53. Redmond ML, Smith S, Collins TC. Exploring African-American womens ' experiences with substance use treatment: a review of the literature. J Community Psychol. 2020;48(2):337-50. Epub 2019/10/15. https://doi.org/10.1002/jcop.22241.

54. Levine AR, Lundahl LH, Ledgerwood DM, Lisieski M, Rhodes GL, Greenwald MK. Gender-specific predictors of retention and opioid abstinence during methadone maintenance treatment. J Subst Abus Treat. 2015;54:37-43. Epub 2015/03/22. https://doi. org/10.1016/j.jsat.2015.01.009.

55. Huhn AS, Berry MS, Dunn KE. Review: sex-based differences in treatment outcomes for persons with opioid use disorder. Am J Addict. 2019;28(4):246-61. Epub 2019/05/28. https://doi.org/10. 1111/ajad.12921.

56. Volkow ND. Stigma and the toll of addiction. N Engl J Med. 2020;382(14):1289-90. https://doi.org/10.1056/NEJMp1917360.

57. Mays VM, Jones AL, Delany-Brumsey A, Coles C, Cochran SD. Perceived discrimination in health care and mental health/substance abuse treatment among Blacks, Latinos, and Whites. Med Care. 2017;55(2):173-81. Epub 2016/10/19. https://doi.org/10.1097/ MLR.0000000000000638.

58. McGinty EE, Barry CL. Stigma reduction to combat the addiction crisis - developing an evidence base. N Engl J Med. 2020;382(14): 1291-2. Epub 2020/04/04. https://doi.org/10.1056/ NEJMp2000227.

59. Fiscella K, Sanders MR. Racial and ethnic disparities in the quality of health care. Annu Rev Public Health. 2016;37:375-94. Epub 2016/01/21. https://doi.org/10.1146/annurev-publhealth-032315021439 .

60. Johnson-Agbakwu CE, Ali NS, Oxford CM, Wingo S, Manin E, Coonrod DV. Racism, COVID-19, and health inequity in the USA: a call to action. J Racial Ethn Health Disparities. 2020. Epub 2020/11/17. https://doi.org/10.1007/s40615-020-00928-y.

61. Drucker E, Anderson K, Haemmig R, Heimer R, Small D, Walley A, et al. Treating addictions: harm reduction in clinical care and prevention. J Bioeth Inq. 2016;13(2):239-49. Epub 2016/04/27. https://doi.org/10.1007/s11673-016-9720-6.

62. Owczarzak J, Weicker N, Urquhart G, Morris M, Park JN, Sherman SG. "We know the streets:" race, place, and the politics of harm reduction. Health Place. 2020;64:102376. Epub 2020/08/26. https://doi.org/10.1016/j.healthplace.2020.102376.

63. MacAfee LK, Harfmann RF, Cannon LM, Kolenic G, Kusunoki Y, Terplan M, et al. Sexual and reproductive health characteristics of women in substance use treatment in Michigan. Obstet Gynecol. 2020;135(2):361-9. Epub 2020/01/11. https://doi.org/10.1097/aog. 0000000000003666.

64. Yang J, Landrum MB, Zhou L, Busch AB. Disparities in outpatient visits for mental health and/or substance use disorders during the COVID surge and partial reopening in Massachusetts. Gen Hosp Psychiatry. 2020;67:100-6. Epub 2020/10/23. https://doi.org/10. 1016/j.genhosppsych.2020.09.004.

Publisher's Note Springer Nature remains neutral with regard to jurisdictional claims in published maps and institutional affiliations. 